\title{
Médiévales
}

Langues, Textes, Histoire

\section{Montanus et les schismatiques : la reprise en main d'une périphérie hispanique au début $\mathrm{du} \mathrm{VI}^{\mathrm{e}}$ siècle}

Montanus and the Schismatics : Retaking Control of a Hispanic Periphery at the Beginning of the 6th Century.

\section{Céline Martin}

\section{CpenEdition}

\section{Journals}

Édition électronique

URL : https://journals.openedition.org/medievales/1586

DOI : $10.4000 /$ medievales. 1586

ISSN : 1777-5892

Éditeur

Presses universitaires de Vincennes

Édition imprimée

Date de publication : 1 décembre 2006

Pagination : 9-20

ISBN : 978-2-84292-193-4

ISSN : 0751-2708

Référence électronique

Céline Martin, «Montanus et les schismatiques : la reprise en main d'une périphérie hispanique au début du vie siècle», Médiévales [En ligne], 51 | automne 2006, mis en ligne le 27 mars 2009, consulté le 22 avril 2022. URL : http://journals.openedition.org/medievales/1586 ; DOI : https://doi.org/ 10.4000/medievales.1586

Ce document a été généré automatiquement le 22 avril 2022.

Tous droits réservés 


\title{
Montanus et les schismatiques : la reprise en main d'une périphérie hispanique au début $\mathrm{du} \mathrm{VI}^{\mathrm{e}}$ siècle
}

\author{
Montanus and the Schismatics : Retaking Control of a Hispanic Periphery at the \\ Beginning of the 6th Century.
}

Céline Martin

1 La collection de conciles visigothique appelée Hispana rassemble quelques rares documents qui ne sont pas des actes conciliaires. Parmi eux, deux lettres non datées de l'évêque Montanus de Tolède, annexées au II ${ }^{\mathrm{e}}$ concile de Tolède de l'année $531^{1}$. Leur seul lien apparent avec les actes de ce concile est leur auteur, qui en a présidé les sessions comme métropolitain, et dont le nom apparait donc dans les souscriptions. Montanus adresse ces deux lettres, au contenu globalement parallèle, respectivement " aux frères et aux fils très aimés du territoire de Palencia ", c'est-à-dire aux clercs palentins, et à un certain Toribius, «mon fils, éminent seigneur et adorateur distingué du Christ» dont la fonction n'est pas précisée. Ces documents posent plusieurs questions d'autant plus gênantes qu'ils se trouvent isolés dans une période d'étiage des sources écrites, que les historiens les ont donc beaucoup sollicités, et souvent interprétés dans des sens contradictoires. Je pense pourtant qu'il est possible aujourd'hui de répondre à ces questions de manière satisfaisante, et de faire ainsi progresser un peu la connaissance des rapports de pouvoir dans la péninsule Ibérique du début du $\mathrm{VI}^{\mathrm{e}}$ siècle ${ }^{2}$.

2 Les lettres de l'évêque Montanus sont des lettres de reproches, adressées néanmoins sur un ton très différent aux clercs palentins et à Toribius. Aux clercs, Montanus commence par rappeler, par une série de citations scripturaires, les châtiments qu'ils encourent s'ils continuent à se comporter en «schismatiques " (le mot apparait deux fois). Il énumère ensuite leurs fautes : de simples prêtres ont consacré le saint chrême, alors qu'ils ont l'obligation d'aller le quérir auprès de leur évêque tous les deux ans ; ils ont invité des évêques «d'un autre ressort» (alienae sortis) pour consacrer des 
basiliques; ils honorent la « secte priscillianiste", non en actes certes, mais au moins en paroles, ce qui doit signifier qu'ils comptent Priscillien (premier hérétique condamné à mort par le pouvoir civil en 385) parmi les martyrs. Désormais, sous peine d'excommunication, et jusqu'à ce que Dieu leur procure "l'évêque accoutumé " (consuetus antistes), il leur faudra l'avertir à temps de leurs besoins soit en saint chrême, soit pour les nouvelles églises, et il y pourvoira lui-même ou par l'un de ses coévêques.

La missive à Toribius commence, elle, par des flatteries : même quand il «fleurissait encore dans le siècle », Toribius était « un ami de la sainte religion ». "Promoteur du culte divin", il a ensuite combattu le priscillianisme, "principalement dans cette province ", et il a travaillé à conduire les "féroces esprits cohabitant avec lui », ceux des "seigneurs terrestres" (terrenorum dominorum), à une "saine règle et à la norme d'une discipline régulière ». Il lui faut pourtant encore, déplore respectueusement Montanus, corriger certains abus dans le territoire de Palencia: face aux prêtres qui consacrent eux-mêmes le chrême, il devra faire usage de « l'autorité sacerdotale la plus sévère " (seuerissimi sacerdotis auctoritate uti); de plus des évêques d'un autre ressort sont invités à consacrer les nouvelles églises; pourtant, dit Montanus, «nous vous envoyons un exemplaire du privilège que votre coévêque a fait pour notre prédécesseur et nos frères les évêques de Carpétanie-Celtibérie, pour que vous sachiez quel fruit pourrait obtenir une demande incorrecte ». Trois municipes ont certes été concédés à ce personnage, mais uniquement tant qu'il vivra, pour ne pas avilir la dignité épiscopale; et si Toribius ne tient pas compte de cet avertissement et prétend enfreindre une "antique coutume", Montanus devra faire appel au roi (domini nostri auribus intimare) et requérir la sentence (districtio) du juge (iudex) Erga, ce qui sera à son détriment. La pietas du roi est en effet telle qu'il ne saurait permettre que l'on change rien à un ius antiquum.

Ces lettres ont d'abord été utilisées pour illustrer la première étape de l'ascension progressive de Tolède au rang de capitale du royaume visigothique ${ }^{3}$, alors même que le roi visigoth réside encore à Narbonne. Ce sont en effet les premiers documents qui attestent l'existence de la Carpétanie-Celtibérie, province nouvelle créée au sein de l'ordonnancement administratif romain et commandée par Tolède, qui dépendait jusque-là de la Carthaginoise et de sa métropole, Carthagène. C'est donc en tant que métropolitain que Montanus réprimande le clergé palentin, et le ius antiquum défendu par le roi goth doit se référer à la hiérarchie ecclésiastique provinciale, qui dans son principe existe depuis un siècle ou un peu moins ${ }^{4}$. La création de la Carpétanie n'est pas due, comme on le lit trop souvent, à l'occupation byzantine du Levant, qui n'a lieu que vingt ans plus tard, en $552^{5}$. Ses limites ne sont pas connues, mais il s'agit visiblement d'une province purement intérieure, s'étendant, autour de Tolède, sur les deux Mesetas. Les lettres de Montanus offrent pourtant plusieurs autres problèmes à résoudre.

Qui est « l'éminent seigneur Toribius»?

5 La première question à soulever est celle de l'identité de Toribius. Les titres que lui décerne Montanus suggèrent qu'il appartient à une famille sénatoriale de Palencia. Il a occupé une position importante dans le monde avant de s'en retirer. L'évolution tardive de l'onomastique romaine ${ }^{6}$ et leur statut social respectif rendent tout à fait vraisemblable l'existence d'un lien de parenté entre Toribius et l'évêque Toribius d'Astorga, avec lequel il a d'ailleurs parfois été confondu - par exemple un lien de grand-père à petit-fils. La région où ils sont actifs est la même : quatre jours de voyage 
séparent Astorga et Palencia ${ }^{7}$. Toribius d'Astorga s'est distingué dans les années 445-450 en affrontant les priscillianistes, dont il a dénoncé les erreurs dans un livre adressé au pape Léon $\mathrm{I}^{\mathrm{er}}$, qu'il connaissait peut-être personnellement ${ }^{8}$. $\mathrm{Au} \mathrm{VI}^{\mathrm{e}}$ siècle une copie de cet écrit, aujourd'hui perdu, se trouve à Palencia: dans sa première lettre Montanus enjoint aux clercs de s'y référer. C'est donc peut-être une tradition familiale qui a poussé Toribius de Palencia à lutter, lui aussi, contre le priscillianisme.

En 531, Toribius n'est plus un dignitaire laïc, il ne «fleurit plus dans le siècle ». Quelque cent trente ans plus tard, Hildefonse de Tolède, dans sa notice sur Montanus ${ }^{9}$, le qualifiera de religiosus, un substantif désignant normalement les moines. Par ailleurs Toribius ne saurait être évêque de Palencia ${ }^{10}$, car les lettres prouvent éloquemment que le siège épiscopal se trouve vacant. Seul un argument pourrait appuyer une telle idée, c'est l'expression uester coepiscopus contenue dans la lettre que lui adresse Montanus: si Toribius a un coévêque, il doit appartenir lui-même à l'épiscopat. Certains manuscrits comportent néanmoins, pour uester, l'abréviation ur ${ }^{11}$, ce qui amène à considérer la possibilité qu'une confusion ait eu lieu, au cours du processus de copie, entre uester et noster. Le personnage en question est en effet, s'il est évêque, un collègue de Montanus, comme les autres suffragants de Carpétanie-Celtibérie qu'il désigne par ce mot ${ }^{12}$.

7 Il ne reste qu'une hypothèse solide : Toribius est l'abbé d'un monastère. À ce titre, il a dû être ordonné prêtre, ce qui lui permettrait de mettre en œuvre face aux clercs «l'autorité sacerdotale la plus sévère », mais n'en fait pas un évêque, bien que le mot sacerdos puisse désigner aussi bien le prêtre que le prélat. Une phrase de Montanus suggère que Toribius appartient bien à l'ordre des moines : «Tu as consacré ta peine à conduire les esprits féroces de ceux qui cohabitaient avec toi à une règle salutaire et à la norme de la discipline régulière ». Cette "cohabitation» pourrait certes faire référence à « l'accommodation » des barbares sur le sol romain, si Toribius est un grand propriétaire foncier - ce qu'il est sans aucun doute. Mais la double mention d'une "règle » incite plutôt à y voir des moines d'origine aristocratique peu enclins à se soumettre à la discipline monastique : ce sont les «seigneurs terrestres", sans autre précision ethnique, dont, selon Montanus, Toribius a corrigé la foi, probablement mêlée de priscillianisme. Rien n'indique en effet qu'il s'agisse de barbares ${ }^{13}$. Depuis le début du v $v^{e}$ siècle les régions hispaniques les plus éloignées de la côte méditerranéenne sont, de fait, indépendantes de Rome, et les puissants locaux, propriétaires fonciers ou évêques, exercent une dominatio que celle des Suèves puis des Goths est simplement venue concurrencer ${ }^{14}$.

8 Ainsi Toribius est-il très probablement un grand personnage du nord de la péninsule qui a fondé un monastère près de Palencia, sans doute dans une villa suburbaine lui appartenant, comme c'est très souvent le cas dans l'Antiquité tardive hispanique ${ }^{15}$. Il s'y est retiré, sans cesser de prêcher contre le paganisme (" par ton instigation l'erreur idolâtre a reculé ») et l'hérésie ( « et la secte détestable et honteuse des priscillianistes a dépéri »). Sa fonction d'abbé et son prestige social en font la plus haute autorité religieuse du territoire palentin, d'autant plus que dans la cité aucun évêque ne lui fait d'ombre. Pourtant on a vu que Montanus évoque, sans le nommer, un « coévêque » qui semble avoir des liens avec Palencia.

Aux origines des désordres : un évêque déposé

9 Montanus n'introduit ce personnage qu'à la fin de la seconde lettre, alors qu'il n'en fait pas mention dans la première. Cette différence entre les deux lettres, le contraste entre 
leur longueur respective et la relative incohérence de la phrase dans laquelle apparait le coepiscopus avaient conduit l'augustin Enrique Flórez ${ }^{16}$ à supposer l'existence d'une lacune dans la version actuelle de la seconde lettre, lacune faisant se court-circuiter deux constructions en licet... tamen ${ }^{17}$. Cette solution semble assez vraisemblable. Malheureusement elle ne dissipe pas toutes les ombres, dans la mesure où le fameux « coévêque » demeure anonyme et où la « demande incorrecte » de Toribius à son sujet n'est pas explicitée.

10 Encore une fois, il faut donc nous livrer au jeu des hypothèses. Notre personnage, que Toribius connaît, est sans nul doute l'ancien évêque de Palencia, car le siège n'est vacant que depuis quelques années. Pour une raison inconnue, il a été déposé par un concile provincial de Carpétanie-Celtibérie antérieur à Tolède II, concile dont il a reconnu la sentence en adressant au prédécesseur de Montanus et aux autres évêques un priuilegium, l'équivalent d'un placitum. Afin de ne pas avilir la dignité épiscopale, le concile lui a cependant concédé trois municipes, Segobia, Brittablo et Cauca. La première et la troisième de ces localités ne posent pas de problème : il s'agit de Ségovie et de Coca, situées à une centaine de kilomètres au sud de Palencia. Brittablo est inconnue. Elle est généralement identifiée à Buitrago, sans motifs solides; ce village se trouve au sud, au-delà de la cordillère du Système central par rapport à Palencia, et la filiation entre les deux noms paraît nébuleuse. Mais une autre identification a été proposée récemment ${ }^{18}$ : celle de Vertavillo, village proche de Palencia (une trentaine de kilomètres au sud), sur la voie romaine Palencia-Clunia, et qui figure dans deux diplômes $\mathrm{du} \mathrm{x}^{\mathrm{e}}$ et $\mathrm{du} \mathrm{xI}^{\mathrm{e}}$ siècle comme Bretavellos et Bertabello. Cette localisation de Brittablo est satisfaisante géographiquement, car la portion détachée du diocèse de Palencia en gagne une certaine cohérence. Il est même possible que l'ex-évêque de Palencia se soit établi à Brittablo, à proximité de son ancien siège épiscopal.

$11 \mathrm{La}$ « demande incorrecte » de Toribius, qui fait réagir Montanus si violemment, ne peut s'expliquer que si l'on admet qu'il manque un passage de la lettre. Sans doute sollicitet-il de recourir au coepiscopus pour l'administration des ordres majeurs, la consécration des basiliques ou du chrême, tous actes qui ne sont pourtant plus de sa compétence ${ }^{19}$; la copie du priuilegium que Montanus lui envoie aurait ainsi pour objet de prouver l'indignité du prélat déposé, qui est peut-être, au demeurant, un allié de Toribius. Les historiens attribuent généralement sa déposition à une ordination irrégulière ${ }^{20}$, la concession de quelques paroisses et des pouvoirs amoindris n'étant pas sans rappeler un cas semblable de sanction infligée, un siècle plus tôt, à Armentaire d'Embrun par le concile de Riez ${ }^{21}$. Qui portait donc la responsabilité de la consécration anticanonique de l'évêque de Palencia? Pour tenter de répondre à cette question, il faut s'intéresser aux diocèses voisins et aux possibles concurrents qu'affronte Montanus de Tolède.

Une région périphérique au début $\mathrm{du} \mathrm{VI}^{\mathrm{e}}$ siècle

Le quart nord-ouest de la péninsule Ibérique, bien que traversé d'un réseau dense de voies lié aux nécessités de l'exploitation minière, a été beaucoup moins romanisé que le sud et la façade méditerranéenne. Ce sont ces terres que les Romains abandonnent en premier aux barbares qui ont franchi les Pyrénées en 409: par le foedus de 411 les Suèves et les Vandales Asdingues se partagent la province de Galice. Une vingtaine d'années plus tard les Vandales quittent l'Hispanie pour l'Afrique, permettant ainsi l'expansion des Suèves, dont la chronique d'Hydace de Chaves ${ }^{22}$ rapporte les incursions jusqu'en Bétique et dans la vallée de l'Èbre. Mais en 456 les Visigoths interviennent en Hispanie et leur infligent de sérieux revers. En 469, quand le récit d'Hydace 
s'interrompt, les Suèves sont divisés, affaiblis, mais tiennent toujours une bonne partie de la province de Galice, y compris, certainement, Astorga ${ }^{23}$. À partir de cette date, et pendant un siècle, nous ne savons absolument plus rien sur eux; ils ne sortent de l'obscurité documentaire qu'en 559, avec leur conversion de l'arianisme au catholicisme sous l'impulsion de Martin de Braga ${ }^{24}$. Parallèlement, des groupes de Visigoths s'implantent progressivement en Hispanie depuis le milieu du $v^{e}$ siècle, le transfert de population s'accélérant après la défaite de Vouillé de 507 ; mais le royaume visigothique, sous tutelle ostrogothique, reste dirigé à partir de la Narbonnaise jusqu'à l'assassinat d'Amalaric en $531{ }^{25}$, sans doute quelques mois après la tenue du $\mathrm{II}^{\mathrm{e}}$ concile de Tolède.

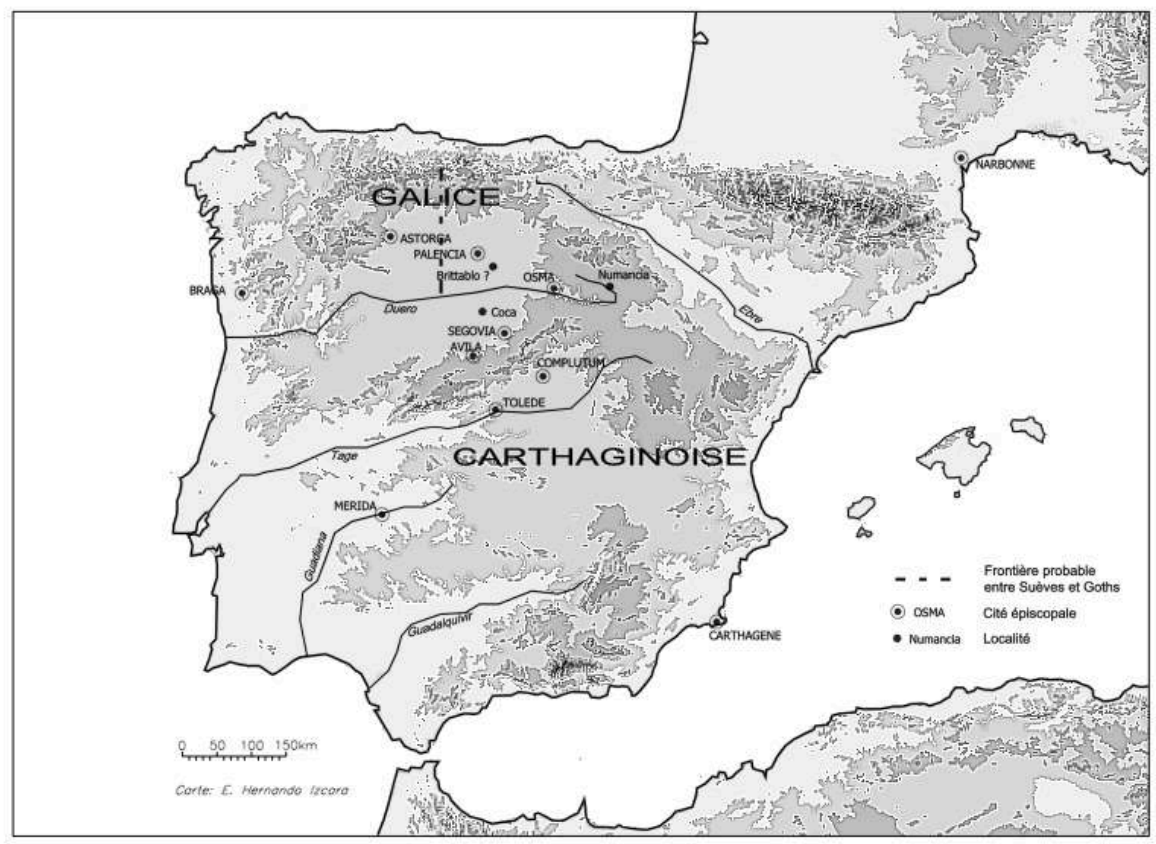

13 Ainsi, dans les années où Montanus et Toribius correspondent, la frontière séparant le regnum sueborum et le regnum gothorum, grossièrement orientée nord-sud, passe quelque part entre Astorga et Palencia. Les historiens sont incapables de la situer précisément, même si certains n'hésitent pas à la décrire comme un limes ${ }^{26}$. Cette incapacité, certes décevante, est peut-être significative: les frontières ne prennent alors qu'exceptionnellement l'aspect de lignes fortifiées, et le mot limes, qui désigne à partir de la fin du $\mathrm{III}^{\mathrm{e}}$ siècle une démarcation militaire frontalière commandée par un $d u{ }^{27}$, est en ce sens révélateur. Même l'appui de la frontière sur un fleuve, traditionnel depuis l'époque romaine, ne signifie pas que l'on n'ait pas affaire à une zone de confins : les Romains n'hésitaient pas à exercer leur imperium au-delà de telles limites, commodes, mais purement théoriques ${ }^{28}$. Dans l'Antiquité tardive, la frontière, loin d'être linéaire, a généralement deux dimensions ${ }^{29}$.

Le territoire de Palencia constitue donc un espace frontalier au contact du royaume suève. Il est de surcroît éloigné des centres de pouvoir, le roi visigothique se trouvant à Narbonne et la région, peu urbanisée, comptant encore peu d'évêques (on ne peut y situer avec certitude que le siège épiscopal d'Astorga, à l'ouest, celui d'Avila, au sudouest, et au sud Complutum, plus près de Tolède que de Palencia); on ne sait rien sur l'éventuelle présence de comtes ou d'autres dignitaires civils. Il s'agit donc d'une zone 
de marge, aux confins des dominations visigothique et suève, et elle l'est d'autant plus qu'elle vient de changer d'obédience. En effet, le territoire de Palencia appartenait depuis le Iv siècle à la province de Galice, tout comme les Asturies, la Cantabrie et le bassin du Duero jusqu'à sa source (Numantia) ${ }^{30}$. La Galice était séparée de la Carthaginoise, province à laquelle appartenait Tolède, par la chaîne du Système central, qui passe au sud de Ségovie et de Coca. Au moment de leur plus grande expansion, c'est-à-dire avant 470 , les Suèves ont sans doute dominé Palencia, Coca et Osma sur le Duero ${ }^{31}$, c'est-à-dire toute la partie orientale de la Galice, échue au départ aux Asdingues. En revanche, au moment où Montanus écrit à Toribius et au clercs palentins, c'est le pouvoir visigothique qui en a pris le contrôle, sans doute dès avant la fin du v siècle.

Dans un tel contexte politique, l'expression alienae sortis episcopi utilisée par Montanus, traduite littéralement ci-dessus par "des évêques d'un autre ressort», semble beaucoup plus claire: contrairement à ce que j'ai pu écrire ${ }^{32}$, il est parfaitement vraisemblable que ces évêques "étrangers" invités à consacrer des basiliques à Palencia viennent de la province de Galice, à laquelle la cité appartenait jusqu'à une époque récente. Le mot sors, qui ne signifie pas regnum, est néanmoins parfaitement indiqué pour désigner deux aires de domination respectivement soumises à deux peuples barbares différents : on se souvient que l'installation des barbares en Hispanie a pris la forme d'une répartition des provinces par tirage au sort ${ }^{33}$. Les évêques « intrusifs » aux yeux de Montanus sont très certainement des prélats des régions suèves voisines, les familles les plus puissantes étant implantées des deux côtés de la nouvelle frontière. Astorga est le siège le plus proche et le premier qui vient à l'esprit : l'éventualité d'un rapport de parenté entre Toribius d'Astorga et Toribius de Palencia est un exemple des liens entre les deux cités. Mais Montanus parle de plusieurs évêques d'une autre sors, ce qui pose problème, car aucun autre évêché suève n'est attesté à l'est de la Galice avant la fin du vi ${ }^{e}$ siècle. Il faut sans doute admettre qu'il existait, en Galice, plus d'évêques que de cités épiscopales, et que, le manque de sources aidant, ils échappent à notre regard. Ce n'est pas une supposition gratuite : la christianisation de la Galice, largement assurée par les priscillianistes aux IV-Ve siècles, a entraîné la consécration d'évêques dans de simples municipes. Les hérétiques considéraient que toute communauté de fidèles, même hors des cités, devait être dirigée par un évêque, personnage auquel ils conféraient un rôle de pasteur ascético-mystique ${ }^{34}$.

Nous retrouvons ainsi le priscillianisme, hérésie dont les lettres de Montanus attestent qu'elle était, encore récemment, implantée à Palencia, et ceci nous ramène à la question de l'élection anticanonique de son ancien évêque. Une réponse se présente d'elle-même: les prélats auxquels les clercs palentins font appel pour consacrer les basiliques, en l'absence d'évêque dans la cité, sont sans doute ceux-là même qui ont procédé à l'ordination irrégulière sanctionnée ensuite par leurs collègues de Carpétanie. Peut-être la consécration avait-elle eu lieu sans l'accord du métropolitain, peut-être aussi un ou plusieurs des prélats consécrateurs étaient-ils suspectés de priscillianisme. La déposition du coepiscopus, la lutte contre le priscillianisme et la réaffirmation des frontières provinciales apparaissent en tout cas fortement liées entre elles.

La reprise en main d'une périphérie

17 Les clercs palentins que Montanus qualifie de "schismatiques " semblent pécher, si toutes ces hypothèses sont correctes, à la fois par déviance religieuse et par déloyauté 
politique. Leur mépris de la hiérarchie ecclésiastique, qui les conduit à s'adjuger des fonctions réservées aux évêques et à ne pas avoir recours à leur métropolitain dans une situation de vacance épiscopale, peut être rattaché à des tendances priscillianisantes, ou même, plus simplement, à des habitudes prises dans un contexte de totale atomisation du pouvoir. L'invocation de Priscillien et de ses disciples au nombre des martyrs correspond à une tradition locale, "galicienne " au sens large : à la fin du IV siècle Ambroise de Milan, en réponse à la consultation de l'évêque Symphosius d'Astorga, lui enjoignait de cesser de les citer parmi les martyrs à la messe. $\mathrm{Au} \mathrm{VI}^{\mathrm{e}}$ siècle le nord de l'Hispanie ne possède pas encore de reliques de martyrs, l'invention de ceux de León datant du siècle suivant : la sanctification peu regardante de Priscillien venait sans doute répondre à un véritable besoin ${ }^{35}$. Enfin, selon Montanus, le fait d'inviter des prélats du royaume suève "nuit aux privilèges de la province et aux intérêts de notre seigneur ${ }^{36}$, autrement dit du roi : le lien étroit qui unit la trame ecclésiastique et l'organisation politique, toutes deux en cours de constitution, est ici manifeste.

C'est ce lien qui permet à Montanus d'informer les clercs de ce que « le bruit en est déjà parvenu aux oreilles du roi », et de menacer plus précisément Toribius d'un précepte royal ou d'une condamnation judiciaire : un éventuel recours au juge sera certainement fondé sur une accusation de trahison. Si Amalaric intervient pour appuyer le pouvoir métropolitain de Montanus, la raison n'en est pas la consolidation de l'influence de Tolède, qui n'est ni capitale ni même encore résidence royale; la défense de la foi catholique contre l'hérésie ne saurait non plus être prise en compte ici, puisque Amalaric est arien. Mais l'Église arienne est certainement très peu développée en Hispanie, peut-être même quasiment inexistante ; les seules structures sur lesquelles peut s'appuyer la royauté visigothique, en situation de grande faiblesse dans les premières décennies $\mathrm{du} \mathrm{VI}^{\mathrm{e}}$ siècle, pour prendre possession d'un territoire encore largement incontrôlé, sont les structures ecclésiastiques catholiques. Quoi qu'en dise Montanus, Amalaric et son entourage ne défendent pas la prééminence de Tolède par pietas, c'est-à-dire par respect de la tradition, mais parce que Montanus, et peut-être Erga, qui pourrait bien être le fonctionnaire visigothique (ou ostrogothique) établi à Tolède pour le nord de la Péninsule, représentent les principales autorités face au royaume suève.

On pourrait même aller plus loin, et proposer une dernière hypothèse : la province de Carpétanie n'a pas été créée, on l'a dit, sous le coup de l'invasion byzantine ; la montée d'une « identité particulière chez les hommes de l'intérieur de la Meseta » est, elle, une explication qui laisse plutôt dubitatif ${ }^{37}$. Il est par contre tout à fait possible qu'elle ait été motivée par le voisinage suève. Le partage de la province de Galice entre deux sortes rendait nécessaire la création d'une nouvelle structure, du côté visigothique, pour éviter toute ingérence de la part de Braga, et Tolède apparaissait comme une métropole certes nouvelle (l'innovation n'est jamais bien vue dans l'Antiquité tardive), mais plus appropriée que Carthagène ou Tarragone, toutes deux très éloignées de ces terres de l'intérieur.

Les lettres de Montanus constituent finalement, au prix de quelques hypothèses, des documents très éclairants sur l'organisation du pouvoir dans le nord de l'Hispanie visigothique au début $d u v^{e}$ siècle. Dans une zone de contact avec la Galice suève, la principale autorité est visiblement aux mains d'un religieux originaire d'une grande famille locale, et probablement lié à des personnages implantés de l'autre côté de la frontière, dans la région d'Astorga. Le pouvoir visigothique, par l'intermédiaire de 
l'évêque catholique de Tolède et d'un délégué civil nommé Erga, tente de reprendre le contrôle de cette marge : c'est le sens de la création d'une nouvelle province, la Carpétanie, et du mélange de flatteries et de menaces adressées à Toribius, afin d'en faire un intermédiaire local fiable face au pouvoir suève.

\section{NOTES}

1. Colección canónica hispana, t. IV, G. MARTìnEZ Dì̀z, F. RodRìGueZ éds., Madrid, 1984, p. 356-366.

2. J'ai déjà examiné ces sources il y a quelques années, dans un article (C. MARTiN, «Las cartas de Montano y la autonomía episcopal de la Hispania septentrional en el siglo VI ", Hispania Antiqua, 22, 1998, p. 403-426) dont je reprends ici certaines idées de départ. Je tiens à remercier les participants au séminaire de Stéphane Lebecq à Lille, où j'ai pu présenter ce dossier, et tout spécialement Frédéric Buche, à qui revient l'idée féconde d'un lien de parenté entre les deux Toribius.

3. A. BARBERO, « Las divisiones eclesiásticas y las relaciones entre la Iglesia y el Estado en la España de los siglos VI y VI », dans ID., La sociedad visigoda y su entorno histórico, Madrid, 1992, p. 168-198.

4. D. MANSILLA, « Orígenes de la organización metropolitana en la Iglesia española », Hispania sacra, XII, 1959, p. 255-290.

5. Erreur déjà réfutée par J. F. Rivera Recio («El encubramiento de la sede toledana durante la dominación visigoda », Hispania Sacra, VIII, 1955, p. 3-34) puis par A. Barbero (cf. n. 3).

6. M. HeinzeLmanN, « Les changements de la dénomination latine à la fin de l'Antiquité », dans Famille et parenté dans l'Occident médiéval, G. DUBY, J. LE GOFF éds., Rome, 1977, p. 19-24.

7. Si l'on suit la moyenne de $45 \mathrm{~km}$ par jour pour un voyageur ordinaire que propose

R. CHEVALLIER, Les voies romaines, Paris, 1972. Sur les voies romaines du nord de

l'Hispanie, voir J. M. Novo GüISAN, Los pueblos vasco-cantábricos y galaicos en la antigüedad tardía. Siglos III-IX, Alcalá de Henares, 1992.

8. Il fut peut-être son secrétaire. Cf. C. TORRES RODRİGUEZ, El reino de los suevos, La Corogne, 1977.

9. HILDEFONSE DE TOLÈdE, De Viris Illustribus, C. CODOÑER éd., El De Viris Illustribus de Ildefonso de Toledo. Estudio y edición crítica, Salamanque, 1972.

10. Certains historiens ont affirmé le contraire, suivant trop rapidement la lecture d'E. A. Thомpson, The Goths in Spain, Oxford, 1969, p. 33, n. 5.

11. Colección canónica hispana, t. IV, op. cit., p. 365, 1. 272.

12. La consécration des églises devra ainsi être accomplie « [...] soit par nous, soit par l'un de nos frères et coévêques selon ce qui nous paraîtra bon » (Ibid., p. 361, 1. 208-209). 13. Ferox, qui peut certes évoquer des barbares, signifie aussi insolent, orgueilleux, ce qui parait une traduction plus exacte que « féroce » pour qualifier les esprits de moines 
aux pratiques ascétiques extrêmes, dans la tradition priscillianiste, et refusant la " discipline régulière ».

14. P. C. DìAZ, « La ocupación germánica del valle del Duero: un ensayo interpretativo », Hispania Antiqua, 18, 1994, p. 457-476.

15. ID., Formas económicas y sociales en el monacato visigodo, Salamanque, 1987.

16. E. FLÓREZ, España sagrada, Madrid, 1750, t. V, p. 418.

17. «Et licet sit in toto orbe sponsae Christi thalamus unus eiusque antistites una in eodem sint fibula caritatis et fidei unione conexi, [*] quid tamen in priuilegium decessori nostro necnon dominis et fratribus nostris Carpetaniae uel Celtiberiae episcopis uester coepiscopus fecit, in exemplaribus caritati uestrae direximus ut scire possitis, improba petitio qualem potuisset habere profectum. " (Colección canónica hispana, t. IV, G. MARTìnEZ DìEZ, F. RODRÌGUEZ éds., Madrid, 1984, p. 365.) La lacune se situerait au lieu marqué d'une astérisque.

18. F. R. GoRdaliza Aparicio, J. M. CANAl SÁnChEZ-PAGÌn, Toponimia palentina, Palencia, 1993.

19. En Occident, les évêques dégradés par des conciles reçoivent parfois le statut de chorepiscopus ou évêque rural, subordonné à l'évêque de la cité, et n'ayant que le pouvoir de conférer les ordres mineurs. Cf. ISIDORE DE SÉVILLE, De Ecclesiasticis Officiis Libri Duo, II, 6, C. M. LAWson éd., CC SL, 113, Turnhout, 1989.

20. C. CODOÑER, El De Viris Illustribus de Ildefonso de Toledo, op. cit., p. 62.

21. Concile de Riez (a. 439), c. 3, dans Concilia Galliae (314-506), Ch. MUNIER éd., CC SL, 148, Turnhout, 1963, p. 63.

22. Hydace, Chronicon, A. Tranoy éd., Hydace. Chronique, SC 218-219, Paris, 1974.

23. P. C. DìAZ, « La ocupación... ", loc. cit.

24. ISIDORE DE SÉVILLE, Historia Gothorum Vandalorum et Sueborum, 90-91, C. RodRìGUEZ ALonso éd., Las Historias de los Godos, Vandalos y Suevos de Isidoro de Sevilla, León, 1975.

25. M. R. VALVERDE CASTRO, Ideología, simbolismo y ejercicio del poder real en la monarquía visigoda: un proceso de cambio, Salamanque, 2000, p. 116-124.

26. L. A. GARCÌA MORENO, «Zamora del dominio imperial romano al visigodo. Cuestiones de historia militar y geopolítica ", dans Actas del I Congreso de Historia de Zamora, t. II, Zamora, 1990, p. 455-465.

27. Le mot limes n'a jamais, avant Mommsen, désigné une frontière linéaire fortifiée. Cf. la mise au point déjà ancienne, mais généralement ignorée des médiévistes, de B. ISAAC, « The Meaning of the Terms Limes and Limitanei », Journal of Roman Studies, 78 (1988), p. 125-147.

28. P. TROUSSET, «La frontière romaine et ses contradictions ", dans La frontière, Travaux de la Maison de l'Orient - 21, Lyon, 1993, p. 25-33.

29. C. MARTIN, « In confinio externis gentibus. La percepción de la frontera en el reino visigodo », Studia Historica. Historia Antigua, 16 (1998), p. 267-280.

30. C. TORRES RODRÌGUEZ, "Límites geográficos de Galicia en los siglos IV y V », Cuadernos de Estudios Gallegos, 4 (1949), p. 367-383. Les auteurs, abusés par la géographie ecclésiastique $\mathrm{du} \mathrm{VII}^{\mathrm{e}}$ siècle, ont longtemps cru que toute cette région appartenait dès l'origine à la Carthaginoise, " précurseur » de la Castille ; aujourd'hui cependant ce point est admis, même par Alain Tranoy, qui a corrigé sa position initiale dans La Galice romaine. Recherches sur le Nord-Ouest de la Péninsule ibérique dans l'Antiquité, Paris, 1981, p. 402-403. 
31. C. TORRES RODRÌGUEZ, "Límites », loc. cit.

32. C. MARTIN, « Las cartas », loc. cit.

33. HYDACE, Chronicon, op. cit., XLIX.

34. M. C. DìAZ Y DìAZ, « Early christianity in Lugo », Classical Folia, 32, 1978, p. 243-259.

J. M. Novo Güisán (op. cit.) distingue, dans le nord de l'Hispanie, une moitié occidentale où la christianisation a été le fait d'évêques, et une moitié orientale où elle est due aux moines. Selon lui la limite passerait par Astorga.

35. C. CARDELLE DE HARTMAN, «Ortodoxos y priscilianistas en la época sueva », dans

Suevos-Schwaben. Das Königreich der Sueben auf der Iberischen Halbinsel (411-585), E. KOLLER, H. LAITENBERGER éds., Tübingen, 1998, p. 81-104.

36. Colección canónica hispana, t. IV, op. cit., p. 361, 1. 204-205.

37. L. A. GARCìA MORENO, "Los orígenes de la Carpetania visigoda », dans Toledo y

Carpetania en la Edad Antigua, Simposio 6-8 de noviembre de 1986, Tolède, 1990, p. 229-249.

\section{RÉSUMÉS}

Une nouvelle lecture des lettres de Montanus de Tolède à Toribius et aux clercs de Palencia (v. 531) suggère que Toribius est un grand personnage devenu abbé qui appuie l'ancien évêque, déposé de son siège. En tant que métropolitain, Montanus tente de dénouer les solidarités traditionnelles de la région avec le reste de la province de Galice, aux mains des Suèves. Cette affirmation de la hiérarchie ecclésiastique sert le roi goth, qui peine à s'affirmer dans ces marges éloignées de Narbonne.

Montanus and the Schismatics: Retaking Control of a Hispanic Periphery at the Beginning of the 6th Century. A re-reading of the letters of Montanus of Toledo to Toribius and to the clergy of Palencia suggests that Toribius is an important personage who has become an abbot and who supports the previously deposed bishop. In his role of metropolitan bishop, Montanus tries to untangle the traditional solidarity of the region with the rest of the province of Galicia that is under the control of the Suebi. This affirmation of the ecclesiastical hierarchy serves the interests of the Gothic king, who has problems in imposing his authority in those borders, remote from Narbonne.

\section{INDEX}

Mots-clés : Barbares, Espagne, pouvoir, priscillianisme

Keywords : Barbarians, power, Priscillianism, province, Spain

\section{AUTEUR}

\section{CÉLINE MARTIN}

Université Lille III-Charles-de-Gaulle, UMR 8529 IRHIS, BP 60149, 59653 Villeneuve-d'Ascq Cedex 